\title{
Nonlinear mean reversion in stock prices: evidence from Asian markets
}

\author{
Kian-Ping Lim* and Venus Khim-Sen Liew \\ Labuan School of International Business and Finance, Universiti Malaysia \\ Sabah, P.O. Box 80594, 87015 F. T. Labuan, Malaysia
}

Utilizing the standard linearity test of Luukkonen et al. (1988), the linear nature of all the Asian stock indices has been formally rejected. This finding warrants use of the nonlinear stationary test of Kapetanois et al. (2003), which is also constructed in the STAR framework, to investigate the mean reverting property of the stock prices series. As a whole, this study not only found convincing evidence of a nonlinear mean reverting pattern in all the Asian stock indices, but also demonstrates the risk of drawing the wrong inferences on mean reversion when the ADF test is applied to data governed by nonlinearity.

\section{Introduction}

Over the past few decades, there has been an increasing interest in the financial economics literature in addressing the issue of mean reversion in stock market prices. Some studies focus on the possibility of returns predictability by investigating whether stock prices follow random walk or mean reversion processes (see, for example, Lo and MacKinlay, 1988; Chaudhuri and $\mathrm{Wu}, 2003,2004)$. Another strand of studies is motivated by the implication of mean reversion property on investment strategies and decisions. Generally, this group of studies proceeds to demonstrate that the detected mean reversion leads to profitable contrarian investment strategy (see, for example, Balvers et al., 2000; Nam et al., 2002).

Earlier work on mean reversion has mainly focused on the US stock market and the findings are inconclusive. Fama and French (1988) and Poterba and Summers (1988) found evidence of mean reversion in stock prices. Lo and MacKinlay (1988), however, found that the stationary mean reverting models could not account for the departures of weekly US returns data from the random walk. Other studies, like Kim et al. (1991) and Richardson (1993), are sceptical and found that earlier work has overstated the evidence of mean reversion. Lately, more effort has been dedicated to addressing the mean reversion phenomenon across national stock markets (Balvers et al., 2000; Chaudhuri and $\mathrm{Wu}, 2003,2004)$.

Most of the aforementioned studies in the literature have utilized regression, variance ratio or unit root tests that are constructed in the linear framework, to examine mean reversion of stock prices. However, there is increasing empirical evidence of nonlinearity in stock prices (for a review of the literature, refer to Lim and Hinich, 2005). Liew et al. (2003), among others, argued that estimating the linear model, implicitly disregarding any possible nonlinearity in the series under consideration, will yield a mis-specified model and thereby provide incorrect inferences.

\footnotetext{
*Corresponding author. E-mail: kianping@ums.edu.my
} 